Investigación experimental

o metaanalítca

Pensar en Movimiento:

Revista de Ciencias del Ejercicio y la Salud

EISSN 1659-4436

Vol. 12, No.1, pp. 1- 23

Cierre al 31 de junio, 2014

\title{
INTERFERENCIA CONTEXTUAL EN EL DESEMPEÑO DE DESTREZAS MOTRICES: UN METAANÁLISIS
}

\section{CONTEXTUAL INTERFERENCE IN MOTOR SKILL PERFORMANCE: A META-ANALYSIS}

\author{
Judith Jiménez Díaz, M.Sc. ${ }^{1(A, B, C, D, E)}$; Walter Salazar Rojas, Ph. D. ${ }^{1(B, D, E)}$; María \\ Morera, Ph.D. ${ }^{2(D, E)}$ \\ judith.jimenez_d@ucr.ac.cr \\ ${ }^{1}$ Escuela de Educación Física y Deportes, Universidad de Costa Rica, Costa Rica \\ ${ }^{2}$ Escuela Ciencias del Movimiento Humano y Calidad de Vida, Universidad \\ Nacional, Costa Rica
}

Manuscrito recibido: 28/06/2013; reenvíos: 26/11/2013, 04/12/2013; aceptado: 19/03/2014;

publicado: 07/05/2014

\section{Resumen}

Jiménez-Díaz, J.; Salazar-Rojas, W. y Morera, M. (2014). Interferencia contextual en el desempeño de destrezas motrices: Un metaanálisis. Pensar en Movimiento: Revista de Ciencias del Ejercicio y la Salud, 12 (1), 1-23. El objetivo del presente metaanálisis es integrar los resultados de los diferentes estudios y examinar posibles variables moderadoras en el uso de la práctica aleatoria y en bloque para la adquisición y retención de destrezas motrices. Por consiguiente, se ubicaron 75 estudios completos publicados en diversas bases de datos electrónicas especializadas en el área, de los cuales se utilizaron 25 estudios. Asimismo, se obtuvo un tamaño de efecto global de $0,34(p \leq 0,05)$, procedentes de 150 tamaños de efecto y un total de 1.256 sujetos. Los tamaños de efecto obtenidos presentaron una alta heterogeneidad, por lo que se evaluaron 12 variables moderadoras. Tanto la práctica en bloque como la aleatoria presentan un tamaño de efecto significativo sobre el desempeño, pero no presentaron diferencias significativas entre sí; lo que sugiere que ambas son igualmente efectivas para mejorar el desempeño de la destreza. Además se encontró diferencia significativa en el momento de la 
adquisición y retención. El desempeño en la adquisición presenta un tamaño de efecto significativo, no así el tamaño de efecto de la retención. Por lo tanto, se concluye que existe una mejora en el desempeño luego de las sesiones de práctica, la cual es moderada por el momento en que se realiza la medición de la destreza. Es necesario aclarar, que para poder concluir con certeza, que la mejora del desempeño se debe a la práctica realizada, se recomienda incluir grupo control en las investigaciones del área.

Palabras claves: práctica aleatoria; práctica en bloque; aprendizaje motor; destreza motriz; estudio metaanalítico.

\begin{abstract}
Jiménez-Díaz, J.; Salazar-Rojas, W. y Morera, M. (2014). Contextual Interference in Motor Skill Performance: A Meta-analysis. PensAR en Movimiento: Revista de Ciencias del Ejercicio y la Salud, 12 (1), 1-23. The purpose of the present meta-analysis is to integrate the results of the different studies and examine possible moderator variables in the use of random and blocked-practices for the acquisition and retention of motor skills. Seventy-five (75) complete studies published in different electronic databases specialized in the area were found, of which 25 were used; an overall effect size of 0.34 ( $p \leq 0.05)$ was derived from 150 effect size (ES) and a total of 1256 subjects. The obtained ES presented high heterogeneity; therefore 12 moderator variables were assessed. Both random practice and blocked-practice presented a significant ES for performance, but had no significant differences among them. This suggests that both are equally effective to improve skill performance. A significant difference was found in the performance of the skill at the moment of acquisition and retention. Acquisition performance presents a significant ES; nevertheless, retention does not. It is concluded that there is an improvement of performance after the practice sessions, which is moderated by the moment when the skill is measured. To be able to state that performance is improved due to the practice, the inclusion of a control group in all experimental studies is recommended.
\end{abstract}

Keywords: random practice; blocked- practice; motor learning; motor skills; meta-analytic study

El orden de enseñanza de las destrezas durante la práctica afecta el desempeño en la adquisición y retención de una destreza durante su aprendizaje (Shea y Morgan, 1979), es por esto que el profesional en Ciencias del Movimiento Humano busca establecer el método más eficiente para la enseñanza de destrezas motrices (Granda-Vera, BarberoÁlvarez, \& Montilla-Medina, 2008; Mazzardo, 2004).

La práctica aleatoria y en bloque son dos técnicas de programación y composición utilizadas para estructurar las experiencias de aprendizaje; las cuales presentan características opuestas de interferencias durante la práctica física, a esto se le llama Interferencia Contextual. Con la aplicación de esta técnica se pretende generar un cambio 
relativamente permanente en la capacidad de movimiento de una persona con base en la práctica -denominado aprendizaje motor- (Schmidt \& Wrisberg, 2008; Shea \& Wright, 1997).

La práctica aleatoria, como su nombre lo dice, propone una secuencia de cierto número de destrezas en un orden aleatorio, mientras que la práctica en bloque es un ensayo de la misma destreza de forma repetitiva (Brady 1998; Schmidt \& Wrisberg, 2008). El Efecto de la Interferencia Contextual ocurre cuando se presenta un mejor aprendizaje de la destreza al utilizar práctica aleatoria, en comparación con la práctica en bloque (Magill \& Anderson, 2013).

Dos hipótesis evalúan este efecto: Hipótesis de la Elaboración -propuesta por Shea y Morgan en 1979- e Hipótesis de Reconstrucción del plan de acción -propuesta por Lee y Magill en 1985-. La primera menciona que al realizar práctica aleatoria la persona utiliza más y diferentes estrategias de la ejecución del movimiento, y al comparar las variantes practicadas puede elaborar una mejor representación de la destreza en su memoria. Por otro lado, la segunda hipótesis plantea que la persona olvida el plan de acción de ejecución de la destreza debido a la alta interferencia ocasionada por las variantes, por lo que debe reconstruir un plan de acción para cada intento (Magill \& Anderson, 2013).

Diferentes autores mencionan que la edad, la habilidad de la persona y la complejidad de la destreza interactúan con el uso de la técnica de Interferencia Contextual (Brady, 2004; French, Rink, \& Werner, 1990), para lograr una mejora en el desempeño de las destrezas. En contraste, hay autores que consideran que el efecto real de diversas variables en el desempeño motor, no ha sido investigado profundamente (Jarus \& Goverover, 1999; Mazzardo, 2004).

El Efecto de Interferencia Contextual ha generado en los últimos años gran cantidad de investigaciones, en las cuales se han encontrado variedad de resultados. En algunos, los autores indican que la práctica aleatoria (Interferencia Contextual alta) perjudica o reduce la adquisición de una destreza, pero mejora la retención y transferencia de la misma, mientras que la práctica en bloque (Interferencia Contextual baja) favorece la adquisición de la destreza, pero reduce la retención y transferencia de la misma (Bertollo, Berchicci, Carraro, Comani, \& Robazza, 2010; Rendell, Masters, Farrow, \& Morris, 2011).

Por otro lado, hay estudios en los cuales los autores mencionan que el tipo de práctica realizada no afecta la mejora del desempeño (Geok, Lay, Grove, Medic, \& Razman, 2012; Zetou, Michalopoulou, Giazitzi, \& Kioumourtzoglou, 2007).

La técnica metaanalítica surge bajo la necesidad de resumir e integrar de forma rigurosa y precisa un conjunto de investigaciones (Botella y Gambara, 2002), entonces se puede resumir como una revisión de literatura estadística que promedia las diferencias entre las condiciones experimentales para proveer un resumen estadístico de confiabilidad y efectividad de diferentes condiciones a lo largo de los estudios (Fontana, Mazzardo, Furtado, \& Gallagher, 2009).

Recientemente se han realizado dos metaanálisis con el fin de determinar el efecto del Modelo Interferencia Contextual en la mejora del desempeño de destrezas motrices, 
ambos en el año 2004. Brady (2004) obtuvo 137 tamaños de efecto, para un efecto global de 0,38, luego de analizar 61 estudios; mientras que Mazzardo (2004) analizó 22 estudios y obtuvo 146 tamaños de efecto.

Debido a la controversia presentada en los resultados en el área de Interferencia Contextual, el presente estudio busca: 1. integrar los resultados de los estudios realizados y 2. examinar posibles variables moderadoras, en el uso de la práctica aleatoria y en bloque, además de conocer su efecto en el desempeño motor de diversas destrezas motrices.

\section{METODOLOGÍA}

\section{Procedimientos de búsqueda}

Una búsqueda de literatura se realizó por computadora en las siguientes bases de datos: EBSCOHost (el cual incluye: Academic Search Premier, Education Research Complete, ERIC, Fuente Académica, MedicLatina, MEDLINE, Academic Search Complete, SocINDEX with Full Text), Google Scholar, Sportdiscus y SpringerLink. Donde se utilizaron como palabras claves: "random practice", "blocked practice", "contextual interference", "motor skill" y "fundamental motor skills", estos términos fueron utilizados tanto de forma individual como combinada.

Adicionalmente, se realizó una búsqueda específica de las referencias identificadas en los artículos relevantes. Un artículo se localizó en la revista impresa de "Perceptual Motor Skills". Asimismo, se recolectaron 96 artículos y/o resúmenes publicados en el área de Interferencia Contextual. Cuando no se encontró el artículo completo en las bases de datos electrónicas o en revistas impresas se contactó al autor (a) principal; de esta manera se consiguieron cuatro artículos completos. En total se contó con 75 artículos completos (ver figura 1).

La búsqueda se efectúo entre setiembre del año 2012 y abril del 2013. No se restringió el rango de la fecha de publicación de los estudios.

\section{Criterios de inclusión/exclusión}

Para que el estudio sea incluido en el metaanálisis se establecieron los siguientes criterios:

1. El artículo debe estar publicado

2. El estudio debe presentar un diseño experimental

3. Se debe evaluar una destreza motriz

4. El diseño debe incluir un grupo de práctica en bloque o al azar

5. La publicación debe presentar promedios y desviaciones estándar 
El estudio que no presentaba uno o más de estos criterios se excluyó del metaanálisis (los estudios excluidos están enumerados en el apéndice 1). Finalmente, se incluyeron 25 estudios, los cuales presentaron los cinco criterios de inclusión.

\section{Procedimiento de codificación de las variables moderadoras}

Las variables moderadores se clasificaron en tres grupos para facilitar la codificación: a. las características de los participantes, b. las características del diseño y c. las variables independientes del estudio. A continuación se enumeran las variables moderadoras del metaanálisis:

1. Características de los participantes:

1. Edad: se codificó el promedio de la muestra en años cumplidos (variable continua).

2. Sexo: hombres, mujeres o mixto (variable categórica).

3. Habilidad del participante: se recopiló como experto o novato (variable categórica).

2. Características de diseño:

1. Validez interna: se construyó una escala para evaluar puntos clave de la validez interna de un estudio (criterios de calidad); la escala consta de cinco ítems: 1. el estudio se describe como aleatorizado, 2. se describe el proceso de aleatorización y es adecuado, 3. el estudio contó con grupo control, 4. hubo mediciones pretest y postest y 5 . se reporta la existencia y manejo de la mortalidad experimental. Se adjudica un "1" si la característica está presente en el estudio y un "0" si no está presente (variable continua).

2. Validez externa: se codificó si el estudio se realizó en el campo o en el laboratorio (variable categórica).

3. Cantidad de intentos: se identificó el total de intentos realizados por los participantes durante el estudio (variable continua).

4. Total de minutos de práctica: se recopiló el total de minutos que duró la práctica (variable continua).

5. Tiempo para medir la retención: cantidad de días entre el postest de adquisición y la medición de retención (variable continua).

3. Características de las variables independientes:

1. Tipo de destreza motriz: se seleccionó como motora gruesa o motora fina (variable categórica).

2. Clasificación de la destreza: se identificó si la destreza es discreta, en serie o continua (variable categórica).

3. Condición de la práctica: se recopiló en práctica en bloque o aleatoria (variable categórica).

4. Momento de la medición: se codificó el momento de medición en adquisición o retención (variable categórica). 
En una búsqueda inicial en bases de datos electrónicas con la utilización de las palabras clave se obtuvo un total de 5.265 referencias

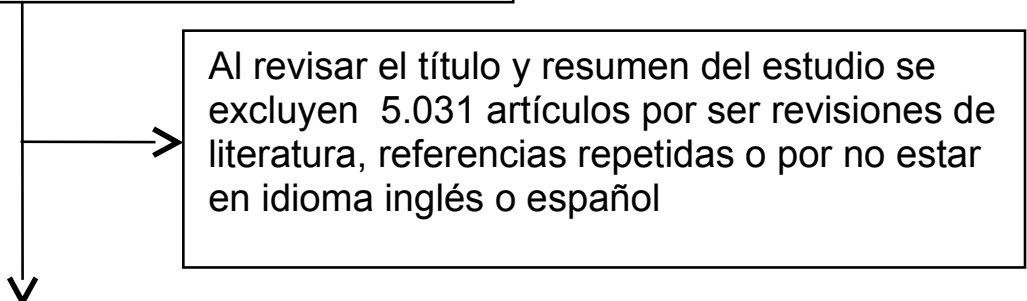

Se identifican 234 artículos potenciales en base de datos electrónicas

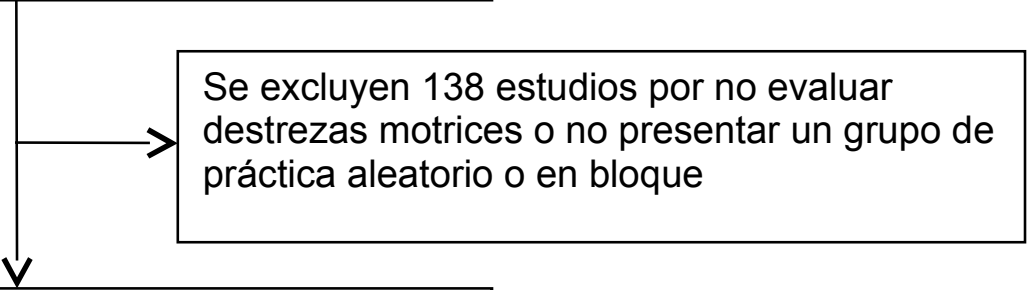

En total se localizan 96 artículos: en bases de datos electrónicas (80), revista impresa (1) y en referencias de artículos completos (13)

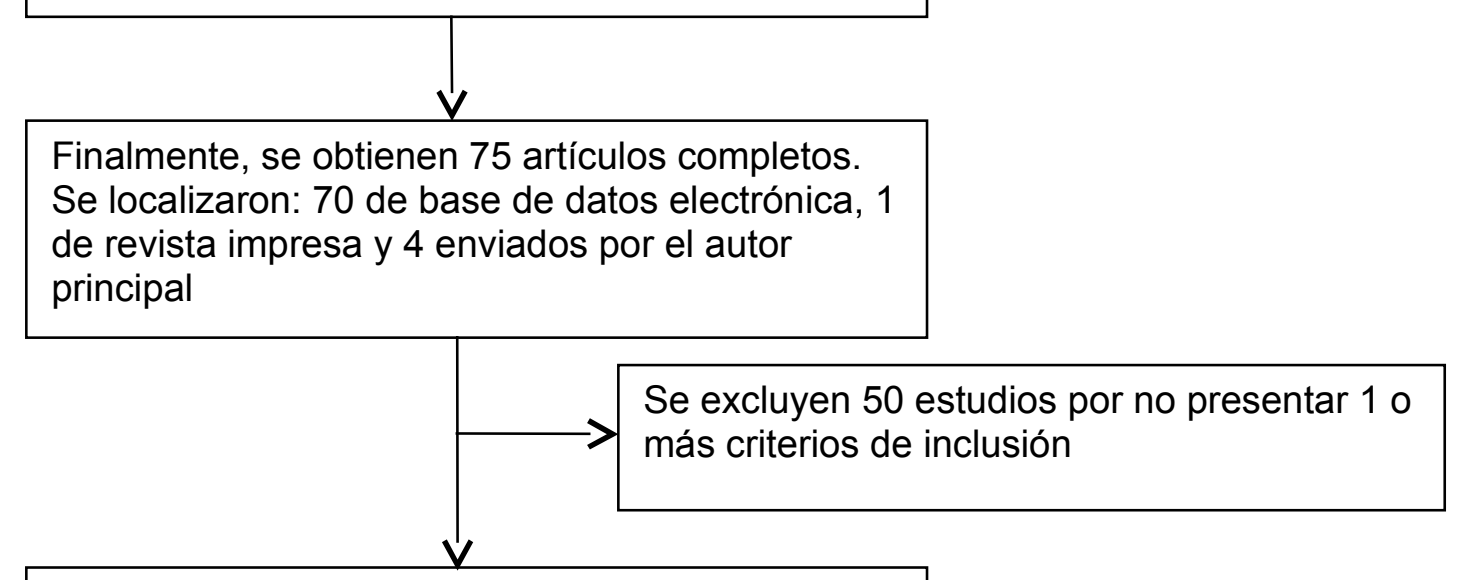

Se incluyen 25 artículos que cumplen con los criterios de inclusión

Figura 1. Flujograma del procedimiento de búsqueda 


\section{Procedimiento del cálculo del tamaño de efecto}

El tamaño de efecto (TE) se obtuvo a lo largo del aprendizaje de la destreza (en el momento de la adquisición y retención, para cada grupo), utilizando la fórmula de delta $(\Delta)$ propuesta por Glass (fórmula 1) (Thomas \& French, 1986).

$$
T E(\Delta)=\frac{M_{1}-M_{2}}{D E_{2}}
$$

Para el cálculo del TE a lo largo del aprendizaje en el momento de adquisición se utilizó el promedio (M) del pretest en $\mathrm{M}_{2}$ y el promedio del momento de adquisición en $\mathrm{M}_{1}$ y la desviación estándar (DE) del pretest. Para el momento de la retención se utilizó el promedio de la adquisición en $\mathrm{M}_{2}$ y el promedio del momento de retención en $\mathrm{M}_{1}$ y la desviación estándar (DE) de la adquisición para cada condición de práctica.

El signo de los tamaños de efecto se ajustó para ser concordante y que un TE positivo indicara beneficio de la práctica realizada. Posteriormente, se aplicó el factor de corrección (c) sugerido por Hedges, utilizando la fórmula 2 (Botella y Gambara, 2002); el cual brinda un TE final calculado. Los TE obtenidos se promedian para obtener el TE global para el grupo control y el grupo experimental.

$$
c=1-\frac{3}{4\left(n_{1}+n_{2}-2\right)-9}
$$

La significancia estadística para el TE global calculado se estableció para la situación experimental y para los TE de los niveles de las variables moderadoras, utilizando intervalos de confianza a un $95 \%$ (ver fórmula $3 \underline{3}$ y $\underline{4}$ ), en donde var(TE) es la varianza de cada tamaño de efecto (Thomas \& French, 1986).

Posteriormente, se realizó el cálculo sugerido por Orwin (1983), con el cual se obtiene la cantidad de tamaños de efecto no significativos necesarios para reducir el TE global obtenido a un tamaño de efecto pequeño.

$$
\begin{array}{r}
I C=T E-1,96 * \sqrt{\operatorname{varTE}} \\
I C=T E+1,96 * \sqrt{\operatorname{varTE}}
\end{array}
$$


La prueba de homogeneidad $Q$ de Cochran's (ver fórmula 5 ), sugerida por Borenstein, Hedges, Higgins y Rothstein (2009), se aplicó con el fin de determinar la heterogeneidad de los TE.

$$
Q=w y^{2}-\frac{(w y)^{2}}{w}
$$

\section{Análisis Estadístico}

La técnica de correlación de Pearson se aplicó para determinar la relación del TE con las variables moderadoras continuas y la técnica de Análisis de Varianza (ANOVA) de una vía, para grupos independientes, de tal modo determinar diferencias entre los TE de los niveles de las variables moderadoras discretas. Para dichos cálculos se utilizó el programa estadístico IBM-SPSS versión $21^{\circledR}$ y el programa de hoja de cálculo de Microsoft Excel ${ }^{\circledR}$.

\section{RESULTADOS}

\section{Tamaño de Efecto Global}

La codificación de los 25 estudios generó un total de 151 tamaños de efecto. De los cuales, 150 son de grupos experimentales y 1 del grupo control, este último no se incluyó en el análisis del estudio, por lo cual se dejó un total de 150 TE. Por consiguiente, se obtuvo un total de 1.256 sujetos. Un TE positivo indica mejora en el desempeño de la destreza.

El tamaño de efecto global, para las condiciones experimentales, inicial fue de 0,35 $(n=150)$, luego de aplicar el factor de corrección se obtuvo un tamaño de efecto global 0,34 (figura 2), el cual es significativamente diferente de 0 (intervalo de confianza 0,27 a $0,40)$.

El cálculo de la prueba de Orwin (1983) brindó como resultado un total de 17 tamaños de efecto no significativos para reducir el tamaño de efecto de 0,34 a un tamaño de efecto de 0,20 .

La prueba $Q$ de Cochran's presenta un valor calculado de 984,44 ( $g l=149 ; p \leq 0,05)$, lo que indica que los tamaños de efectos calculados son heterogéneos.

Al considerar la heterogeneidad de los tamaños de efecto se analizó la presencia de variables moderadoras. Por lo tanto se tomaron en cuenta las siguientes variables moderadoras: edad, sexo, habilidad del sujeto, destreza motora, cantidad de intentos durante la práctica, momento de la medición, condición de la práctica, cantidad de tiempo para medir la retención, validez externa e interna del estudio, algunas de estas sugeridas por Brady (2004) y Mazzardo (2004). 
Tabla 1

Significancia de los tamaños de efectos calculados

\begin{tabular}{|c|c|c|c|c|c|c|}
\hline $\begin{array}{c}\text { Variable } \\
\text { Moderadora }\end{array}$ & $\mathbf{n}$ & TE & IC & $F$ & $\mathbf{r}$ & $p$ \\
\hline Edad & 101 & 0,42 * & 0,33 a 0,50 & & 0,018 & 0,859 \\
\hline Sexo & 142 & 0,35 * & 0,28 a 0,41 & 0,55 & & 0,579 \\
\hline Femenino & 34 & 0,54 * & 0,40 a 0,68 & & & \\
\hline Masculino & 5 & 0,58 * & 0,15 a 1,01 & & & \\
\hline Ambos & 103 & 0,30 * & 0,22 a 0,38 & & & \\
\hline Habilidad del & 144 & 0,36 * & 0,29 a 0,43 & 0,08 & & 0,780 \\
\hline Participante & & & & & & \\
\hline Novatos & 135 & $0,35 *$ & 0,28 a 0,42 & & & \\
\hline Expertos & 9 & 0,48 * & 0,21 a 0,74 & & & \\
\hline Tipo de destreza & 150 & 0,33 * & 0,27 a 0,40 & 3,41 & & 0,067 \\
\hline Motora Gruesa & 127 & 0,42 * & 0,35 a 0,49 & & & \\
\hline Motora Fina & 23 & $-0,15$ & $-0,33$ a 0,03 & & & \\
\hline $\begin{array}{l}\text { Clasificación de la } \\
\text { destreza }\end{array}$ & 150 & 0,34 * & 0,27 a 0,40 & 0,03 & & 0,851 \\
\hline Discreta & 138 & 0,33 * & 0,26 a 0,39 & & & \\
\hline Serie & 12 & 0,40 * & 0,16 a 0,65 & & & \\
\hline Validez Interna & 150 & 0,34 * & 0,27 a 0,40 & & 0,064 & 0,436 \\
\hline Validez Externa & 145 & 0,35 * & 0,29 a 0,42 & 1,37 & & 0,243 \\
\hline Laboratorio & 12 & $-0,09$ & $-0,33$ a 0,14 & & & \\
\hline Campo & 133 & 0,39 * & 0,32 a 0,46 & & & \\
\hline $\begin{array}{l}\text { Cantidad de } \\
\text { Intentos }\end{array}$ & 115 & 0,25 * & 0,17 a 0,32 & & 0,105 & 0,263 \\
\hline $\begin{array}{l}\text { Cantidad de } \\
\text { minutos }\end{array}$ & 26 & 0,52 * & 0,37 a 0,67 & & $-0,133$ & 0,517 \\
\hline $\begin{array}{l}\text { Tiempo para medir } \\
\text { la retención }\end{array}$ & 81 & 0,03 & $-0,05$ a 0,12 & & $-0,201$ & 0,073 \\
\hline $\begin{array}{l}\text { Condición de la } \\
\text { práctica }\end{array}$ & 150 & 0,33 * & 0,27 a 0,40 & 1,79 & & 0,182 \\
\hline Bloque & 82 & 0,19 * & 0,10 a 0,28 & & & \\
\hline Aleatorio & 68 & 0,50 * & 0,40 a 0,60 & & & \\
\hline $\begin{array}{l}\text { Momento de la } \\
\text { medición }\end{array}$ & 150 & 0,33 * & 0,27 a 0,40 & $9,81^{*}$ & & 0,002 \\
\hline Adquisición & 70 & 0,70 * & 0,60 a 0,80 & & & \\
\hline Retención & 80 & 0,01 & $-0,07$ a 0,10 & & & \\
\hline
\end{tabular}

\section{Variables Moderadoras}

Para cada nivel de las variables moderadoras se obtuvo un intervalo de confianza y de esa forma establecer la significancia del TE promedio en cada nivel. Para determinar el efecto de las variables moderadoras se realizaron análisis de correlación (Pearson) o análisis de variabilidad (ANOVA), para variables continuas o discretas respectivamente (ver tabla 1). 


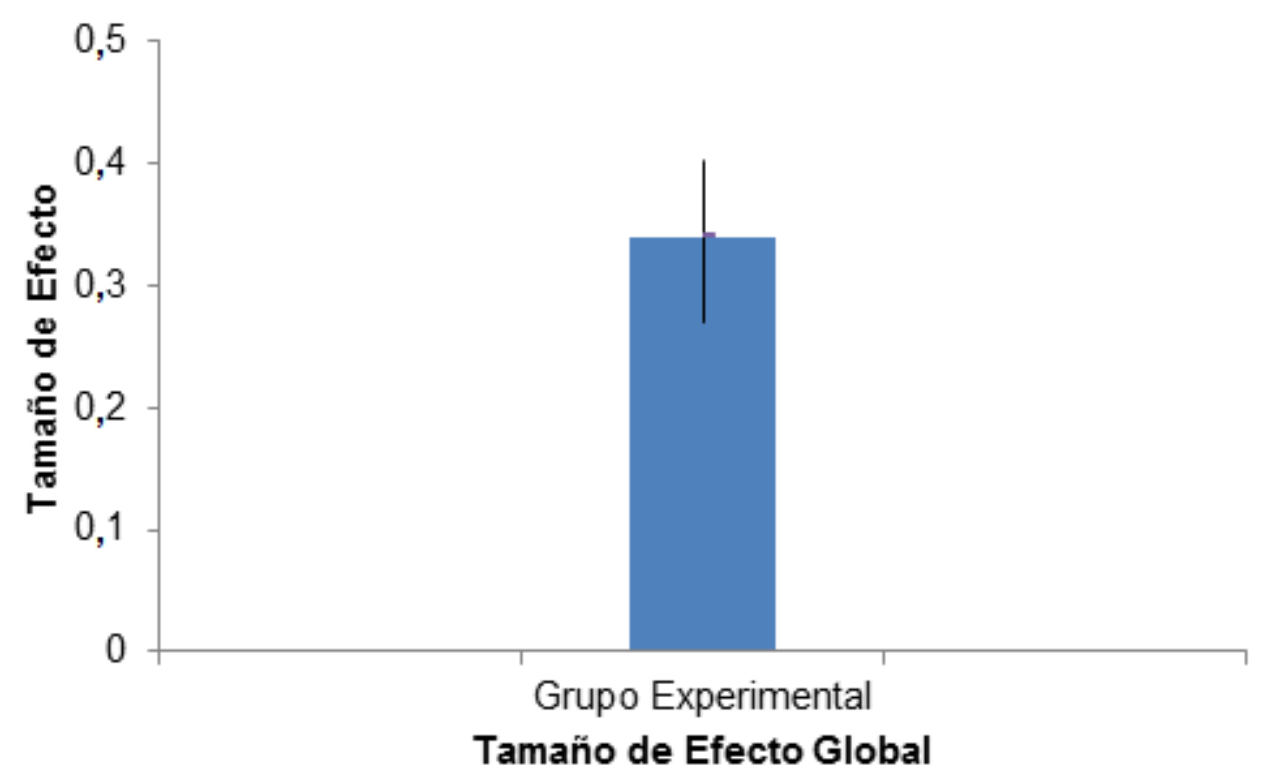

Figura 2. Tamaño de Efecto global corregido para los grupos experimentales.

$\mathrm{TE}=0,34(p \leq 0,05)$

Edad. La figura 3 muestra la correlación de los valores del TE calculado con la edad promedio de los participantes para cada estudio $(n=101)$, se obtuvo una relación no significativa.

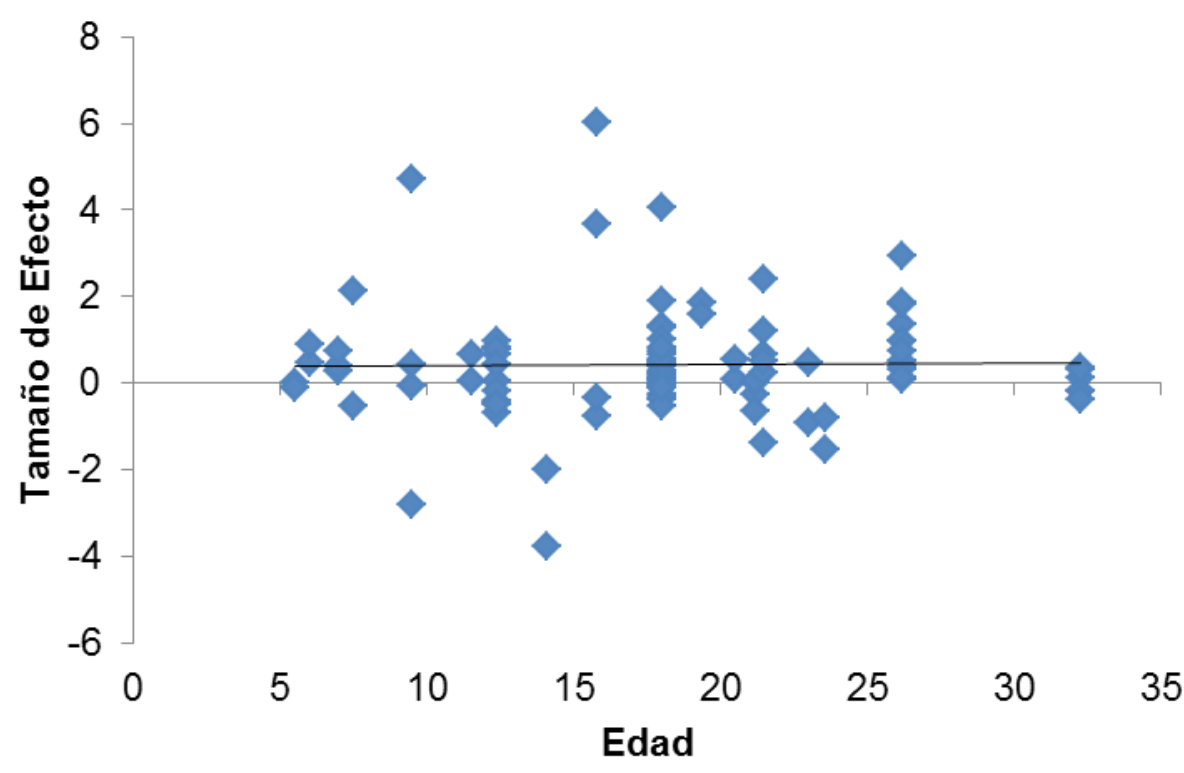

Figura 3. Relación entre la edad de los participantes y el tamaño de efecto, $r=, 018(p=, 859)$. 
Sexo. Cuando se comparó los TE de los estudios en los que solo participaron mujeres $(n=34)$, solo hombres $(n=5)$ o mixto $(n=103)$, no se obtuvo una diferencia estadísticamente significativa entre los grupos (figura 4).

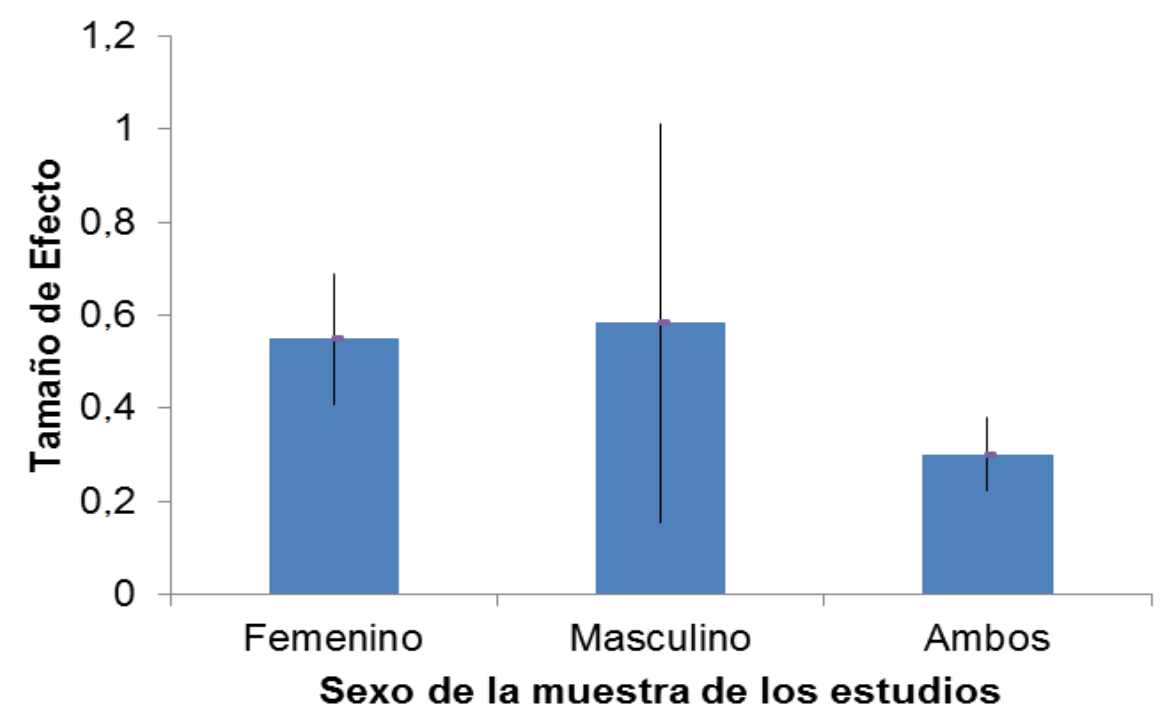

Figura 4. Tamaño de efecto según el sexo de los participantes en el estudio,

$$
F_{(2,139)}=, 549(p=, 579) \text {. }
$$

Habilidad del participante. En este apartado no se ubicó diferencia entre los TE según la habilidad de los participantes (figura 5 ): novatos $(n=135)$ o expertos $(n=9)$.

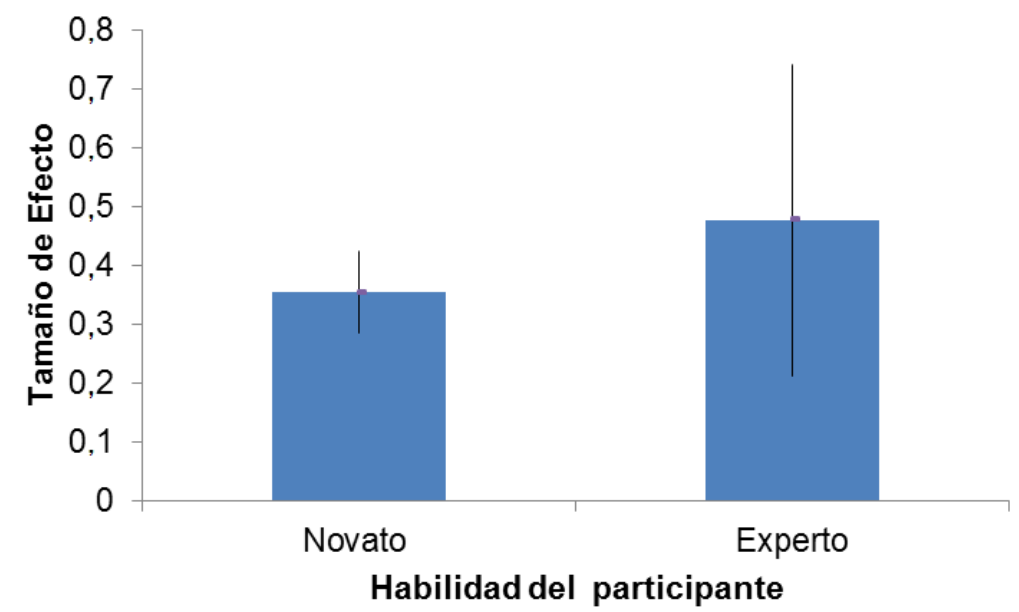

Figura 5. Tamaño de efecto según la habilidad de los participantes en el estudio, $\mathrm{F}_{(1,142)}=$, $078(p=, 780)$. 
Tipo de destreza. Al comparar el tamaño de efecto de los diferentes estudios y considerar el tipo de destreza investigada (figura 6), no se encontró diferencia entre los estudios que involucran motora gruesa $(n=127)$ y destrezas de motora fina $(n=23)$.

Figura 6. Tamaño de efecto según el tipo de destreza evaluada en el

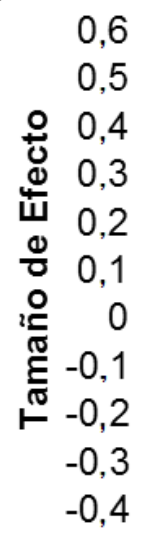

0,6

0,5

0,4

0,3

0,2

0,1

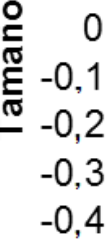

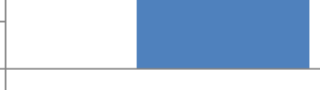

\section{Gruesa \\ Fina \\ Destreza Motora}

estudio, $F_{(1,148)}=3,41(p=, 067)$.

Clasificación de la destreza. En este caso no se presentó diferencia entre los estudios que involucran destrezas discretas $(n=138)$ y destrezas en serie $(n=12)$; ningún estudio presentó destrezas continuas (figura 7).

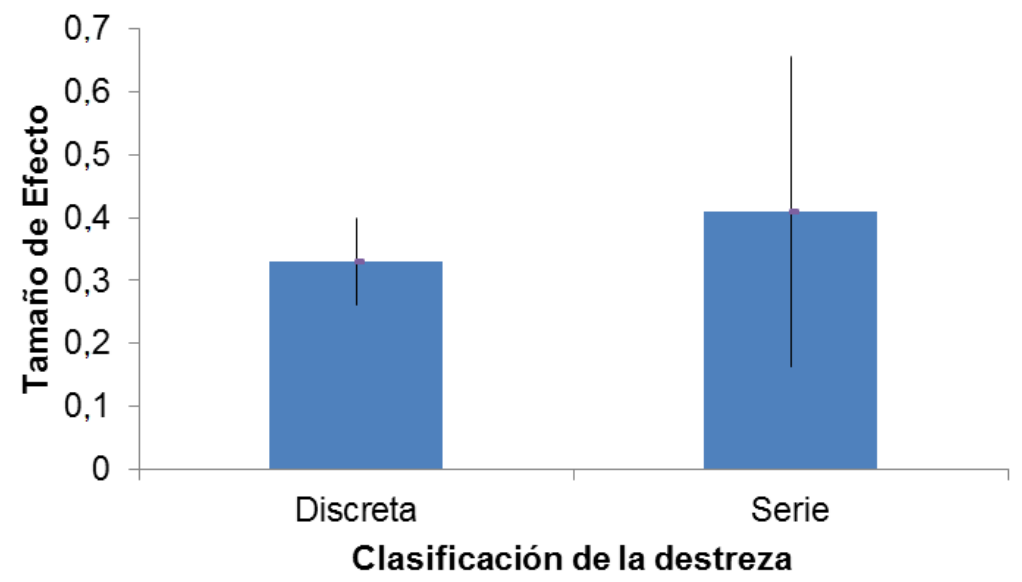

Figura 7. Tamaño de efecto según la clasificación de la destreza evaluada en el estudio, $F_{(1,148)}=, 035(p=, 851)$. 
Validez interna. Al utilizar la escala se encontró que la mayoría de los estudios presentan dos puntos de cinco, los cuales son los ítems uno y cuatro antes mencionados. No se ubicó una relación positiva (figura 8).

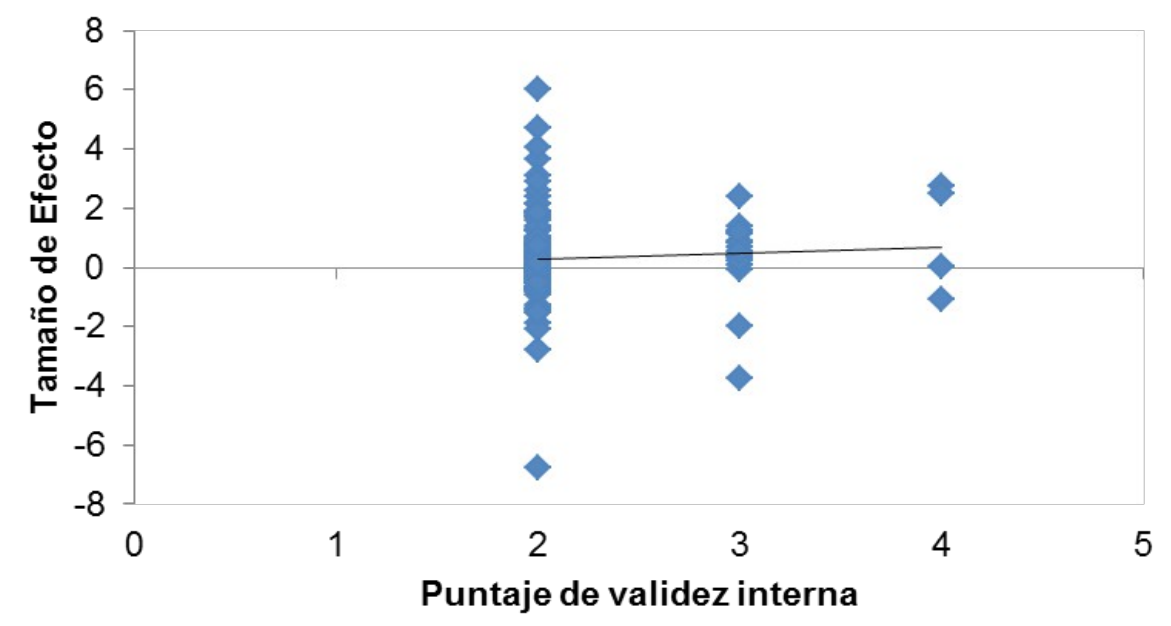

Figura 8. Relación entre el puntaje de validez interna y el tamaño de efecto, $r=, 064(p=, 436)$.

Validez externa. En este apartado no se presentó diferencia entre los estudios que trabajaron en el campo $(n=133)$ y los estudios que trabajaron en el laboratorio $(n=12)$. Ver figura 9.

Figura 9. Tamaño de efecto según la validez externa del estudio,

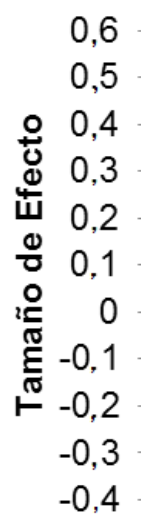


Cantidad de intentos. En la figura 10 se muestra la correlación entre los valores del TE calculado y la cantidad de intentos realizados durante la práctica para cada estudio $(n=115)$, no se obtuvo una relación significativa.

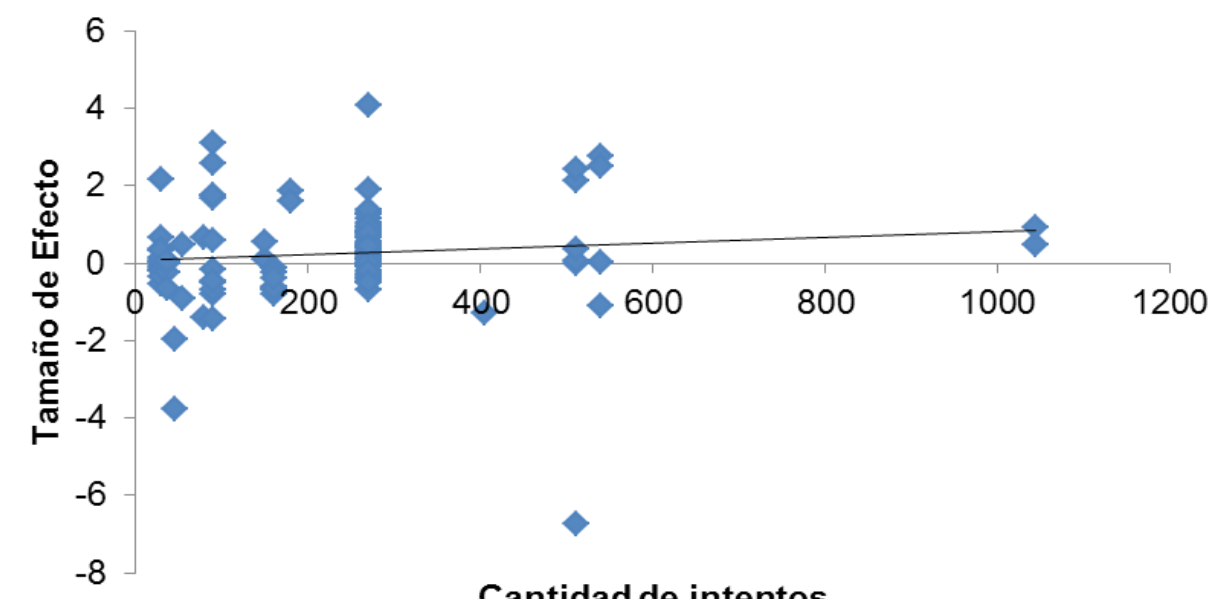

Figura 10. Relación entre la cantidad de intentos durante la práctica y el tamaño de efecto, $r=, 105(p=, 263)$.

Total de minutos de práctica. En este caso no se presentó una relación significativa entre los valores del TE calculado y el total de minutos dedicado a la práctica durante las sesiones experimentales $(n=26)$. Ver figura 11.

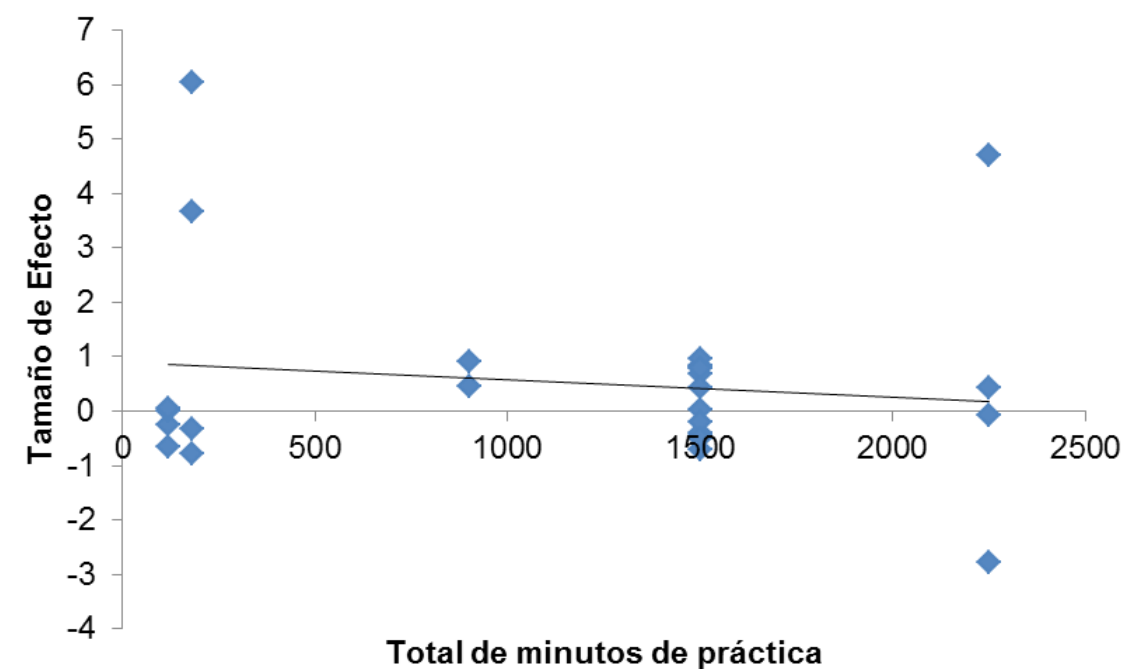

Figura 11. Relación entre la cantidad de minutos durante la práctica y el tamaño de efecto, $r=-, 133(p=, 517)$. 
Tiempo para medir la retención. No se ubicó una relación significativa en el tiempo para medir la retención $(n=81)$. Ver figura 12.

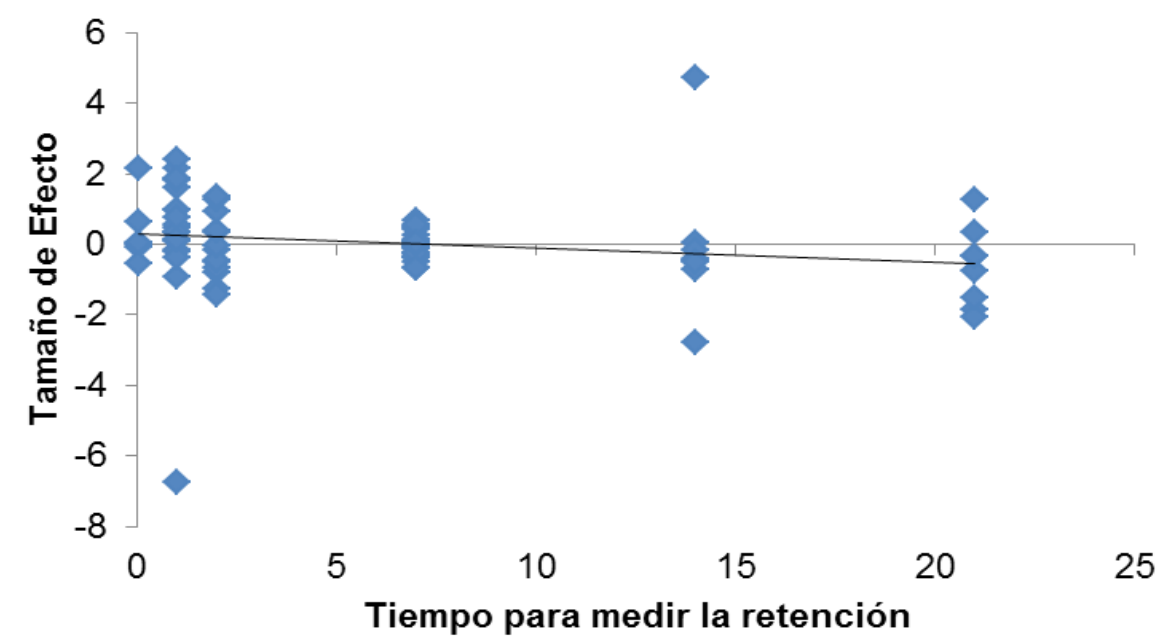

Figura 12. Relación entre los días entre las mediciones de adquisición y retención y el tamaño de efecto calculado para la retención, $r=-, 201(p=, 073)$.

Condición de la práctica. No se encontró diferencia entre la práctica en bloque $(n=82)$ y la práctica aleatoria $(\mathrm{n}=68)$. Ver figura 13.

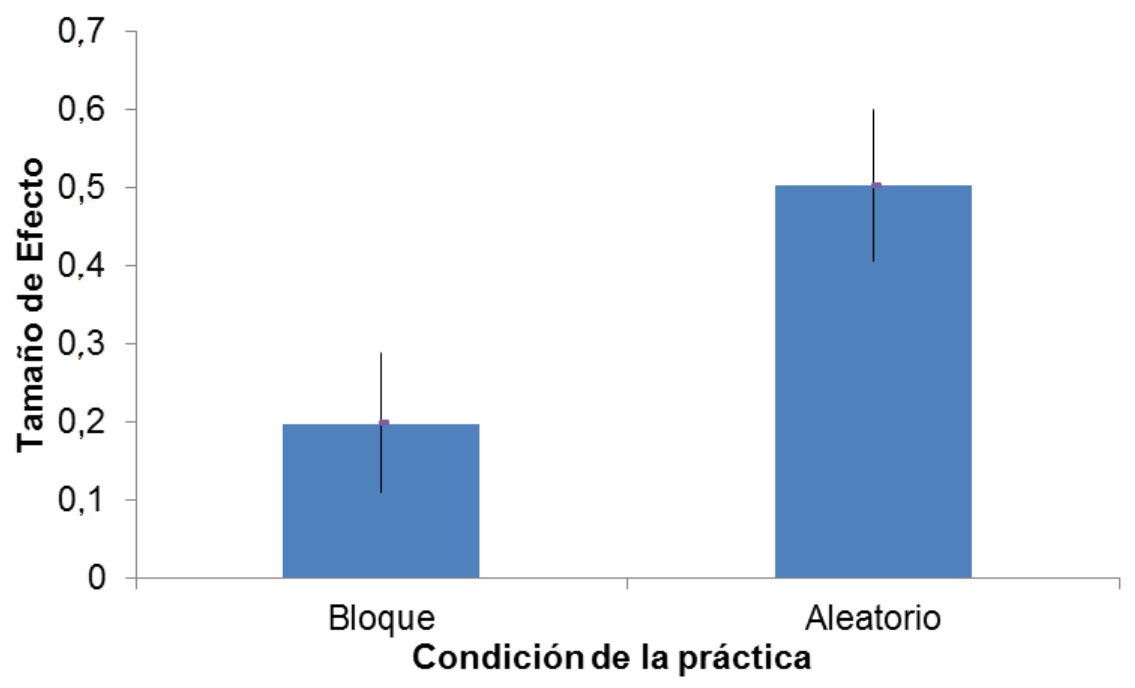

Figura 13. Tamaño de efecto según la condición de la práctica, $F_{(1,148)}=1,79(p=, 182)$. 
Momento de la medición. En este caso (figura 14), se presentó diferencia significativa entre la adquisición $(n=70)$ y la retención de la destreza $(n=80)$.

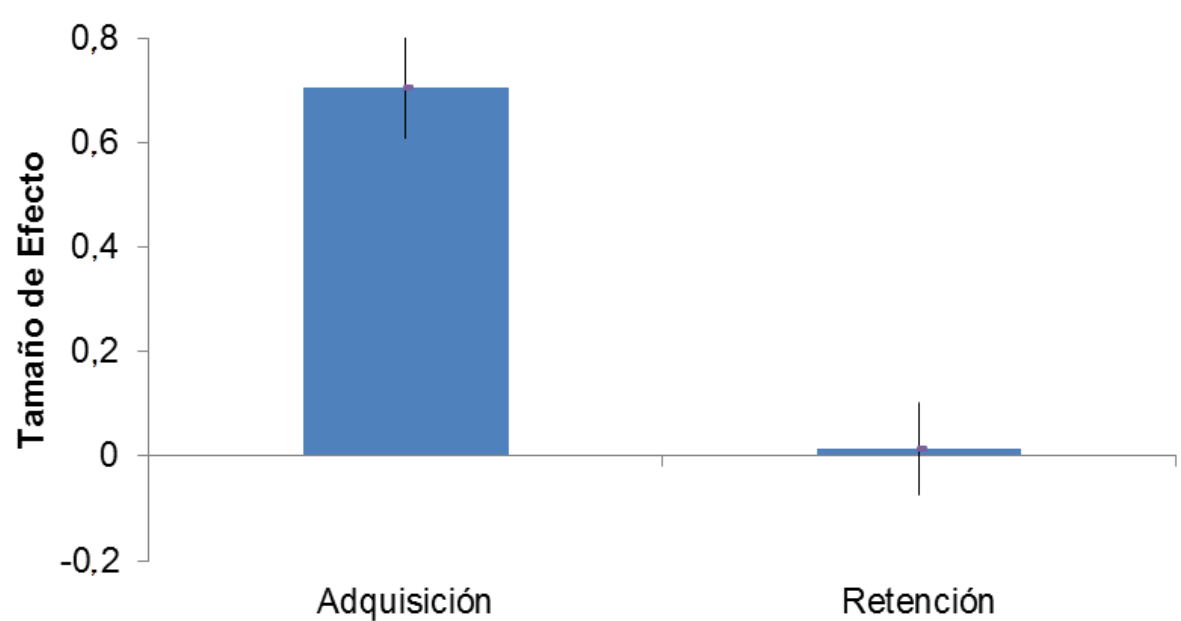

Momento de la medición

Figura 14. Tamaño de efecto según el momento de la medición,

$$
F_{(1,148)}=9,81^{*}(p=, 002) \text {. }
$$

\section{DISCUSIÓN}

Los resultados de este estudio sugieren un efecto global entre pequeño y mediano $(T E=0,34)$ en la mejora del desempeño luego de realizar práctica física. Cabe destacar que un tamaño de efecto (TE) de 0,20 representa un tamaño pequeño, 0,50 representa un tamaño moderado, mientras que 0,80 representa un tamaño de efecto grande (Cohen, 1988). Este tamaño de efecto global se refleja como la mejora del desempeño de forma general. Debido a la variabilidad de los tamaños de efectos individuales se buscó el efecto de variables moderadoras.

Jarus y Goverover (1999) indicaron en su estudio que la edad puede influenciar el efecto de la interferencia contextual, pero el efecto real no ha sido examinado. Al evaluar la variable de edad, no se encontró una relación significativa, este resultado concuerda con lo presentado por Zipp y Gentile (2010), quienes compararon el desempeño de una destreza motora gruesa entre niños(as) y personas adultas, y concluyeron que ambos grupos de edad mejoran su desempeño durante la fase de adquisición y no se encontró influencia de la edad en los cambios del desempeño para la retención. Por lo que la edad del participante no interfiere en la mejora del desempeño de la destreza motriz. 
No es tan común que la muestra de participantes de los estudios sea un grupo de hombres (Porter \& Saemi, 2010) o un grupo de mujeres (Bertollo et al. 2010), lo usual son las muestras en donde se encuentran ambos sexos (Geok et al., 2012; Landin \& Hebert, 1997; Meira \& Tani, 2001); aun así no se ubicaron investigaciones en los que se haya realizado un análisis comparativo entre hombres y mujeres. Por lo tanto, en el presente estudio se incluyó como variable moderadora el sexo de los participantes, variable que no se ha evaluado en otros estudios. Al no presentarse una diferencia entre las muestras de hombres, mujeres o ambos, se sugiere que indistintamente, si el grupo está compuesto por uno o ambos sexos esto no afecta el aprendizaje de la destreza.

Los resultados de este estudio sugieren que indistintamente de la condición de práctica realizada, la habilidad del participante no afecta la mejora en el desempeño. Herbert et al. (1996) encontraron que los estudiantes novatos se benefician más de práctica en bloque, mientras que para los estudiantes con mayor destreza no se presentó diferencia según el tipo de práctica; por su parte Mazzardo (2004), en su metaanálisis, indica que no hay suficiente información para llegar a una conclusión.

Brady (1998) indica que el tipo de destreza es un factor relevante en la mejora del desempeño motor. Por lo tanto, se comparó las destrezas clasificadas en motora gruesa o motora fina y también clasificadas en serie o discretas (no hubo suficiente información para obtener un tamaño de efecto de destrezas continuas); no se encontró diferencia entre ninguna de las clasificaciones. Cuando Mazzardo (2004) clasificó las destrezas en abiertas y cerradas, no pudo establecer comparaciones por la falta de información.

También, se evaluó la validez interna de cada estudio por medio de una escala, no se encontró relación entre el puntaje obtenido en la escala y el tamaño de efecto, por lo que este estudio sugiere que la validez interna del estudio no afecta el tamaño de efecto obtenido del mismo. Aunque se presenta una leve tendencia a una relación positiva, lo que implicaría que cuanta mayor validez interna mayor tamaño de efecto. Estos resultados son similares a los expuestos por Mazzardo (2004), donde encuentra que estudios con mayor control interno presentan tamaños de efecto más robustos.

Hebert et al. (1996) mencionan que el trabajo de campo es usualmente más complejo que el de laboratorio, lo que requiere mayor tiempo para dominar la destreza. Por su parte Brady (2004) indica que los estudios de laboratorio tienden a presentar mayores tamaños de efecto, mientras que los estudios de campo tienden a presentar tamaños de efectos inconsistentes, debido a la cantidad de factores que pueden influir el desempeño, además de la precisión de los instrumentos utilizados en el laboratorio en comparación con los utilizados en el campo. En este estudio no se encontró diferencia entre los dos escenarios -estudios de campo y de laboratorio-, lo cual es contradictorio a lo encontrado por Brady (2004), pero similar a lo reportado por Mazzardo (2004). Lo que indica que ambos grupos de práctica se benefician de ambos escenarios de trabajo.

Al igual que lo concluido por Mazzardo (2004), no se encontró beneficio en el desempeño dado por la cantidad de práctica. Cabe destacar que se presenta una tendencia a obtener un mayor tamaño de efecto al aumentar la cantidad de intentos, no así en el total de minutos de práctica. 
Ningún estudio ha evaluado la diferencia en el desempeño al variar el tiempo para medir la retención. Por esto, se incluyó como variable el tiempo transcurrido para la evaluación de la retención, no se encontró una relación significativa entre el tamaño de efecto de la retención y la cantidad de días de la medición. Los resultados sugieren una tendencia a disminuir el TE entre más tiempo transcurra.

Al comparar la utilización de la práctica en bloque contra la práctica aleatoria, ambos extremos del efecto de interferencia contextual, se presentó una diferencia no significativa entre ambas técnicas. De manera similar, en otros estudios no encontraron diferencias entre el desempeño de la destreza al realizar la práctica en bloque o aleatoria (GrandaVera et al., 2008; Pollatou, Kioumourtzoglou, Agelousis, \& Mavromatis, 1997; Travlos, 2010; Zetou et al., 2010).

Cuando se presentan diferencias debido a la condición de la práctica, estas, aunque sean pequeñas se inclinan a favor de la práctica aleatoria (Brady, 1998), lo que es acorde con la tendencia presentada, en este estudio, por un mayor tamaño de efecto en la práctica aleatoria.

Asimismo, se analizó tanto el tamaño de efecto de la adquisición de la destreza como el de la retención de la misma, al compararlas se determinó que existe diferencia entre ambas mediciones, indistintamente del tipo de práctica realizada la adquisición presenta mayor TE con respecto a la retención. En diversos estudios se ha encontrado beneficios en el desempeño motor, dependiendo de la condición de la práctica en el momento de la medición (Keller, Li, Wiess, \& Relyea, 2006; Pollatou et al, 1997).

El Efecto de Interferencia Contextual propone que el uso de una alta interferencia en la práctica -práctica aleatoria- limita la adquisición de la destreza y favorece la retención, al contrario de utilizar baja interferencia en una sesión de trabajo -práctica en bloque(Brady, 1998). Los resultados de este estudio, al igual que otros estudios (Granda-Vera et al., 2008; Zetou et al., 2010), no apoyan lo presentado por la teoría del efecto de la interferencia contextual. Aun así, indican que indistintamente de la utilización de la interferencia contextual durante la práctica, se mejora el desempeño motor en el momento de la adquisición de la destreza, no así en la retención.

\section{CONCLUSIONES Y RECOMENDACIONES}

En general, los hallazgos de este estudio indican que la edad, el sexo de las muestras de los estudios, la habilidad del participante, el tipo de destreza, la validez (interna o externa) del estudio, la cantidad y la condición de práctica no moderan la mejoría en el desempeño de una destreza motriz, pero sí indican que indistintamente de lo anterior, existe una mejora del desempeño en el momento de la adquisición de la destreza, no así en el momento de evaluar la retención de la misma.

Los resultados de este metaanálisis no apoyan el Efecto de la Interferencia Contextual, el cual indica que se da un mejor aprendizaje de la destreza al realizar 
práctica aleatoria; ya que ambos tipos de práctica (bloque y aleatorio) benefician el desempeño en el momento de la adquisición, no así en la retención.

Cabe destacar, que debido a la falta de estudios que no presentan la información necesaria para el análisis metaanalítico (promedio y desviaciones estándar), no se pudo incluir 50 de 75 estudios. Por lo tanto, se recomiendan realizar más estudios que evalúen diversos factores en el uso de Interferencia Contextual, para obtener conclusiones más contundentes, además de incluir grupo control, para poder determinar con certeza que la mejora en el desempeño (el aprendizaje motor) se debe a la realización de la práctica y no a otros factores que pueden influir en el aprendizaje motor.

\section{REFERENCIAS}

$\left(^{*}\right)$ Estudios utilizados para el metaanálisis

*Bertollo, M., Berchicci, M., Carraro, A., Comani, S., \& Robazza, C. (February, 2010). Blocked and random practice organization in the learning of rhythmic dance step sequences. Perceptual and Motor Skills, 110(1), 77-84. doi: 10.2466/pms.110.1.7784 Ir a artículo http://www.amsciepub.com/doi/abs/10.2466/pms.110.1.77-84

$$
\text { REGRESAR A: } \underline{\text { TEXTO1 }} \text { TEXTO 2 }
$$

*Bortoli, L., Spagolla, G., \& Robazza, C. (August, 2001). Variability effects on retention of a motor skill in elementary school children. Perceptual and Motor Skills, 93(1), 51-63. doi: $\quad 10.2466 / p m s .2001 .93 .1 .51 \mathrm{lr}$ a http://www.amsciepub.com/doi/abs/10.2466/pms.2001.93.1.51

Borenstein, M., Hedges, L.H., Higgins, J.P.T. \& Rothstein, H.R. (2009). Introduction to Meta-Analysis. Wiltshire, Inglaterra: Wiley. Ir a libro: http://www.wiley.com/WileyCDA/WileyTitle/productCd-EHEP002313.html.

$$
\text { REGRESAR A TEXTO }
$$

Botella, J., \& Gambara, H. (2002). Qué es el Meta-análisis. Madrid: Editorial Biblioteca Nueva, S.L. Ir a libro: http://www.casadellibro.com/libro-que-es-el-metaanalisis/9788470309915/835899 REGRESAR A:

TEXTO 1

TEXTO 2

Brady, F. (1998). A Theoretical and Empirical Review of the Contextual Interference Effect and the Learning of Motor Skills. QUEST, 50(3), 266-293. doi: 10.1080/00336297.1998.10484285 Ir a artículo: http://www.tandfonline.com/doi/abs/10.1080/00336297.1998.10484285? journalCode=uqst20\#.U13IKYF5MYI

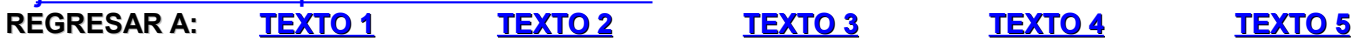

Brady, F. (August, 2004). Contextual interference: a meta-analytic study. Perceptual and Motor Skills, 99(1), 116-126. doi: 10.2466/pms.99.1.116-126 Ir a artículo: http://www.amsciepub.com/doi/abs/10.2466/pms.99.1.116-126

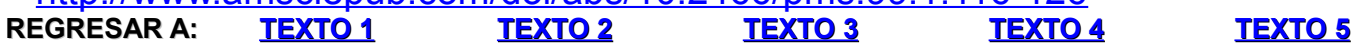

Cohen, J. (1988). Statistical Power Analysis for the Behavioral sciences $\left(2{ }^{\text {nd }}\right)$. Hillsdale: Lawrence Erlbaum Associates, Publicshers. Ir libro: 
http://www.Irdc.pitt.edu/schneider/P2465/Readings/Cohen, \%201988\%20(Statistical \%20Power, \%20273-406).pdf

REGRESAR A TEXTO

Fontana, F. E., Mazzardo, O., Furtado, O., Jr., \& Gallagher, J. D. (October, 2009). Whole and part practice: a meta-analysis. Perceptual and Motor Skills, 109(2), 517-530. Ir a artículo: http://www.amsciepub.com/doi/abs/10.2466/pms.109.2.517-530

\section{REGRESAR A TEXTO}

${ }^{*}$ French, K. E., Rink, J. E., \& Werner, P. H. (August, 1990). Effects of contextual interference on retention of three volleyball skills. Perceptual and Motor Skills, 71(1), 179-186. doi: 10.2466/pms.1990.71.1.179 Ir a artículo: http://www.amsciepub.com/doi/abs/10.2466/pms.1990.71.1.179

\section{REGRESAR A TEXTO}

${ }^{*}$ Geok, J., Lay, B., Grove, J. R., Medic, N., \& Razman, R. (2012). Practicing Field Hockey Skills Along the Contextual Interference Continuum: a Comparison of Five Practice Schedules. Journal of Sports Science and Medicine, 11(2), 304-311. Ir Ir a artículo: http://www.jssm.org/vol11/n2/14/v11n2-14text.php REGRESAR A TEXTO 1 REGRESAR A TEXTO 2

* Goodwin, J. E., \& Meeuwsen, H. J. (December, 1996). Investigation of the contextual interference effect in the manipulation of the motor parameter of over-all force. Perceptual and Motor Skills, 83(3), 735-743. doi: 10.2466/pms.1996.83.3.735Ir a artículo: http://www.amsciepub.com/doi/abs/10.2466/pms.1996.83.3.735

${ }^{*}$ Granda-Vera, J., Barbero-Alvarez, J. C., \& Montilla-Medina, M. (April, 2008). Effects of different practice conditions on acquisition, retention, and transfer of soccer skills by 9-year-old schoolchildren. Perceptual and Motor Skills, 106(2), 447-460. doi: 10.2466/pms.106.2.447-460 Ir a artículo: http://www.amsciepub.com/doi/abs/10.2466/pms.106.2.447-460

\section{REGRESAR A TEXTO 1 REGRESAR A TEXTO 2 REGRESAR A TEXTO 3}

*Granda-Vera, J., \& Montilla-Medina, M. (June, 2003). Practice schedule and acquisition, retention, and transfer of a throwing task in 6-yr.-old children. Perceptual and Motor Skills, 96(3), 1015-1024. doi: 10.2466/pms.2003.96.3.1015 Ir a artículo: http://www.amsciepub.com/doi/abs/10.2466/pms.2003.96.3.1015

*Hall, K. G., Domingues, D. A., \& Cavazos, R. (June, 1994). Contextual interference effects with skilled baseball players. Perceptual and Motor Skills, 78(3), 835-841. doi: $\quad 10.2466 / p m s .1994 .78 .3 .835 \quad$ Ir $\quad$ a http://www.amsciepub.com/doi/abs/10.2466/pms.1994.78.3.835

*Hebert, E. P., Landin, D., \& Solmon, M. A. (1996). Practice schedule effects on the performance and learning of low - and high-skilled students: an applied study. Research Quarterly for Exercise and Sport, 67(1), 52-58. doi:10.1080/02701367.1996.10607925 Ir a artículo: http://www.tandfonline.com/doi/abs/10.1080/02701367.1996.10607925\#.U2EpF_I5 $\underline{P} \underline{E}$

REGRESAR A TEXTO 1 REGRESAR A TEXTO 2

*Jarus, T., \& Goverover, Y. (April, 1999). Effects of contextual interference and age on acquisition, retention, and transfer of motor skill. Perceptual and Motor Skills, 88(2), 
437-447. doi: 10.2466/pms.1999.88.2.437 Ir a artículo: http://www.amsciepub.com/doi/abs/10.2466/pms.1999.88.2.437

REGRESAR A: TEXTO 1 TEXTO 2

*Jones, L. L., \& French, K. E. (2007). Effects of contextual interference on acquisition and retention of three volleyball skills. Perceptual and Motor Skills, 105(3 Pt 1), 883890. doi: 10.2466/pms.105.3.883-890 Ir a artículo: http://www.amsciepub.com/doi/abs/10.2466/pms.105.3.883-890

*Keller, G. J., Li, Y., Weiss, L. W., \& Relyea, G. E. (August, 2006). Contextual interference effect on acquisition and retention of pistol-shooting skills. Perceptual and Motor Skills, 103(1), 241-252. doi: 10.2466/pms.103.1.241-252 Ir a artículo http://www.amsciepub.com/doi/abs/10.2466/pms.103.1.241-252 REGRESAR A TEXTO

*Landin, D., \& Hebert, E. P. (1997). A comparison of three practice schedules along the contextual interference continuum. Research Quarterly for Exercise and Sport, 68(4), 357-361. doi: 10.1080/02701367.1997.10608017 Ir a artículo: http://www.tandfonline.com/doi/abs/10.1080/02701367.1997.10608017\#.U2Ek3vl5 $\underline{P} \underline{E}$

REGRESAR A TEXTO

${ }^{*} \mathrm{Li}$, Y., \& Wright, D. L. (May, 2000). An assessment of the attention demands during random-and blocked-practice schedules. The Quarterly Journal of Experimental Psychology: Human Experimental Psychology, 53A(2), 591-606. doi: $10.1080 / 027249800390628$ Ir a artículo: http://psycnet.apa.org/psycinfo/2000-08368-013

Magill, R., \& Anderson, D. (2013). Motor Learning and Control: Concepts and Applications (10 $0^{\text {th }}$ ed.). New York: McGraw-Hill. Ir a libro: http://mhprofessional.com/product.php?cat=108\&isbn=0078022673

$$
\text { REGRESAR A: TEXTO 1 }
$$

Mazzardo, O. (2004). Contextual Interference: Is it supported across studies? (Tesis de maestría). Recuperada de D-Scholarship Institutional Repository at the University of Pittsburgh. (http://d-scholarship.pitt.edu/9942/)

$\begin{array}{llllll}\text { REGRESAR A: } & \text { TEXTO 1 } & \text { TEXTO 2 } & \text { TEXTO 3 } & \text { TEXTO 4 } & \text { TEXTO 5 } \\ \text { TEXTO 6 } & \text { TEXTO 7 } & \text { TEXTO 8 } & \text { TEXTO 9 } & \end{array}$

*Meira, C., \& Tani, G. (June, 2001). The Contextual Interference Effect in acquisition of dart-throwing Skill Tested on a Transfer Test with Extended Trials. Perceptual and Motor Skills, 92(3), 910-918. Ir a artículo: http://www.amsciepub.com/doi/abs/10.2466/pms.2001.92.3.910 REGRESAR A TEXTO

Orwin, R. G. (1983). A fail-safe $\mathrm{N}$ for effect size in meta-analysis. Journal of Educational Statistics, 8(2),157-159. Ir a artículo: http://www.jstor.org/stable/1164923?seq=1 
*Pollatou, E., Kioumourtzoglou, E., Agelousis, N., \& Mavromatis, G. (April, 1997). Contextual interference effects in learning novel motor skills. Perceptual and Motor Skills, 84(2), 487-496. doi: 10.2466/pms.1997.84.2.487 Ir a artículo: http://www.amsciepub.com/doi/abs/10.2466/pms.1997.84.2.487

\section{REGRESAR A TEXTO 1 TEXTO 2}

*Porter, J., \& Saemi, E. (2010). Moderately Skilled Learners Benefit by Practicing with Systematic Increases in Contextual Interference. International Journal of Coaching Science, 4(2), 61-71.

\section{REGRESAR A TEXTO}

Rendell, M. A., Masters, R., Farrow, D., \& Morris, T. (2011). An Implicit Basis for the Retention Benefits of Random Practice. Journal of Motor Behavior, 43(1), 1-13. doi: 10.1080/00222895.2010.530304 Ir a http://www.tandfonline.com/toc/vjmb20/43/1\#.U2Esw_I5P_E

REGRESAR A TEXTO

Schmidt, R. A., \& Wrisberg, C. A. (2008). Motor Learning and Performance. A SituationBased Learning Approach $\left(4^{\text {th }}\right.$ ed.). Champaign, Illinois: Human Kinetics. Ir a libro: http://books.google.co.cr/books/about/Motor_Learning_and_Performance.html? id=Ejc27Wrg5rMC\&redir esc $=y$

REGRESAR A: IEXTO 1

Shea, C., \& Wright, D. (1997). An: The Sciences of Physical Education: Introduction to Human Movement. Estados Unidos: Benjamin Cummings. Ir a libro: http://catalogue.pearsoned.co.uk/catalog/academic/product?ISBN=0137951132 REGRESAR A TEXTO

Shea, J. B., \& Morgan, R. L. (1979). Contextual Interference Effects on the Acquisition, Retention, and Transfer of a Motor Skill. Journal of Experimental Psychology: Human Learning and Memory, 5(2), 179-187. doi: 10.1037/0278-7393.5.2.179 Ir a artículo: http://psycnet.apa.org/journals/xlm/5/2/179/

$$
\text { REGRESAR A: TEXTO 1 }
$$

*Simon, D. A. (August, 2007). Contextual interference effects with two tasks. Perceptual and Motor Skills, 105(1), 177-183. doi: 10.2466/pms.105.1.177-183 Ir a artículo: http://www.amsciepub.com/doi/abs/10.2466/pms.105.1.177-183

*Smith, P. J. (1997). Attention and the contextual interference effect for a continuous task. Perceptual and Motor Skills, 84(1), 83-92. doi: 10.2466/pms.1997.84.1.83 Ir a artículo: http://www.amsciepub.com/doi/abs/10.2466/pms.1997.84.1.83

*Smith, P. J. (December, 2002). Applying contextual interference to snowboarding skills. Perceptual and Motor Skills, 95(3), 999-1005. doi: 10.2466/pms.2002.95.3.999 Ir a artículo: http://www.amsciepub.com/doi/abs/10.2466/pms.2002.95.3.999

Thomas, J., \& French, K. (1986). The Use of Meta-Analysis in Exercise and Sport: A Tutorial. Research Quarterly for Exercise and Sport, 57(3), 196-204. doi:

10.1080/02701367.1986.10605397 Ir a artículo: http://www.tandfonline.com/doi/abs/10.1080/02701367.1986.10605397\#.U2Emqfl5P E

REGRESAR A: TEXTO 1 TEXTO 2 
*Travlos, A. K. (February, 2010). Specificity and variability of practice, and contextual interference in acquisition and transfer of an underhand volleyball serve. Perceptual and Motor Skills, 110(1), 298-312. doi: 10.2466/pms.110.1.298-312 Ir a artículo: http://www.amsciepub.com/doi/abs/10.2466/pms.110.1.298-312

REGRESAR A TEXTO

*Wegman, E. (February, 1999). Contextual interference effects on the acquisition and retention of fundamental motor skills. Perceptual and Motor Skills, 88(1), 182-187. doi: $\quad 10.2466 / p m s .1999 .88 .1 .182 \quad$ Ir $\quad$ a http://www.amsciepub.com/doi/abs/10.2466/pms.1999.88.1.182

*Wright, D. L., Black, C. B., Immink, M. A., Brueckner, S., \& Magnuson, C. (2004). Longterm motor programming improvements occur via concatenation of movement sequences during random but not during blocked practice. Journal of Motor Behavior, 36(1), 39-50. doi: 10.3200/JMBR.36.1.39-50. Ir a artículo: http://www.tandfonline.com/doi/abs/10.3200/JMBR.36.1.39-50\#.U2Ep1vl5P_E

*Wright, D. L., Magnuson, C. E., \& Black, C. B. (2005). Programming and reprogramming sequence timing following high and low contextual interference practice. Research Quarterly for Exercise and Sport, 76(3), 258-266. doi: 10.1080/02701367.2005.10599297 Ir a http://www.tandfonline.com/doi/abs/10.1080/02701367.2005.10599297\#.U2EmGPI $\underline{5 P E}$

*Zetou, E., Michalopoulou, M., Giazitzi, K., \& Kioumourtzoglou, E. (June, 2007). Contextual interference effects in learning volleyball skills. Perceptual and Motor Skills, 104(3), 995-1004. doi: 10.2466/pms.104.3.995-1004 Ir a artículo: http://www.amsciepub.com/doi/abs/10.2466/pms.104.3.995-1004

$$
\text { REGRESAR A: TEXTO 1 } 1 \text { TEXTO 2 }
$$

Zipp, G. P., \& Gentile, A. (February, 2010). Practice schedule and the learning of motor skills in children and adults: teaching implications. Journal of College Teaching \& Learning, $\quad 7(2), \quad$ Ir $35-42 . \quad$ a artículo: http://journals.cluteonline.com/index.php/TLC/article/view/87/84 REGRESAR A TEXTO

Participación: A- Financiamiento, B- Diseño del estudio, C- Recolección de datos, D- Análisis estadístico e interpretación de resultados, E- Preparación del manuscrito. 